\title{
Estudio evaluativo de riesgo psicosocial en el desempeño de labores de seguridad privada
}

\author{
Evaluation Study of Psychosocial Risk in the Performance of Private Security Work \\ Raquel Muñoz-Hernández'1, Saúl Rangel-Lara² \\ 1 Universidad Politécnica del Valle de México, MÉxIco \\ https://orcid.org/0000-0002-4461-8027 | jael2222@hotmail.com, raquel.munoz@upvm.edu.mx \\ 2Universidad Politécnica del Valle de México, MÉxIco \\ https://orcid.org/0000-0003-1498-340X | industrial@upvm.edu.mx
}

Recibido 13-02-2020, aceptado 17-07-2020.

\begin{abstract}
Resumen
El estudio se realizó con el personal de vigilancia y seguridad en una institución de educación superior del Estado de México, en ella se analizan factores relacionados a un requerimiento normativo que establece los elementos para identificar, analizar y prevenir los factores de riesgo psicosocial, así como para promover un entorno organizacional favorable en los centros de trabajo e incrementar la Productividad en el desempeño del personal de seguridad. El Proyecto se refiere a la sensibilización de la NOM-035-STPS-2018 que fue dada a conocer para consulta pública el 26 de octubre de 2016 y fue publicada el 23 de octubre del 2018, para entrar en vigor el 23 de octubre de 2019. El método estadístico aplicado fue el análisis de regresión entre la productividad en el desempeño administrativo (Variable suma de productos ya que en forma conceptual se expresa como la producción de recursos por unidad de tiempo), de las Variables de la Ergonomía Organizacional y para darle sustento explicativo a las variables, se utiliza una matriz de correlaciones entre las variables y los Método Ergonómicos RULA y OWAS. Los Resultados muestran que existe un alto riesgo para los trabajadores de seguridad de adquirir patologías a mediano y largo plazo.
\end{abstract}

Palabras clave: riesgo, violencia laboral, normatividad.

\begin{abstract}
The study was carried out in an institution of higher education in the State of Mexico, it analyzes factors related to a regulatory requirement that establishes the elements to identify, analyze and prevent psychosocial risk factors, as well as to promote a favorable organizational environment in work centers and increase productivity in the performance of security personnel. The Project refers to the sensitization of NOM-035-STPS-2018 that was released for public consultation on October 26, 2016 and was published on October 23, 2018, to enter into force on October 23, 2019 The Statistical Method applied was the Regression Analysis between productivity in administrative performance (Variable sum of products since in conceptual form it is expressed as the production of resources per unit of time), of the Variables of the Organizational Ergonomics and to give it explanatory support for the variables, a matrix of correlations between the variables and the Rula and OWAS Ergonomic Methods is used. The results show that there is a high risk for security workers to acquire pathologies in the medium and long term.
\end{abstract}

Index terms: risk, workplace violence, regulations. 


\section{INTRODUCCIÓN}

En los últimos años, con los avances tecnológicos, se ha incrementado la cantidad de industrias manufactureras, de servicios y de todo en los sectores de la sociedad a nivel mundial. Esto aumenta por consecuencia las fuentes de trabajo en todas las áreas, incluyendo el personal de seguridad con sus particulares implicaciones en riesgo laboral. La Organización Internacional del Trabajo (OIT) [1], reporta que anualmente ocurren más de 317 millones de accidentes en el trabajo y cada día mueren 6,300 personas a causa de accidentes o enfermedades relacionadas con el trabajo. El coste de esta adversidad diaria es enorme y la carga económica de las malas prácticas de seguridad y salud se estima en un cuatro por ciento del Producto Interno Bruto (PIB), global de cada año, implicando además de las pérdidas humanas, pérdidas financieras. En 2008, la OIT adoptó el Programa de Seguridad y Salud en el Trabajo y Medio Ambiente, que tiene como objetivo crear conciencia mundial sobre la magnitud y las consecuencias de los accidentes, las lesiones y las enfermedades relacionadas con el trabajo y los riesgos disergonómicos.

En la actualidad existe un considerable vacío en la aplicación de la ergonomía y solo se aplica en algunos puestos de trabajo del sector manufacturero, sin considerar que es una herramienta de prevención de accidentes y preservación de la salud de todo tipo de persona que realiza una actividad en el desempeño de sus funciones, contribuyendo a mejorar las condiciones de seguridad y salud en el trabajo y por consecuencia en el incremento de la productividad.

El presente estudio se desarrolla en una institución de educación superior pública en el estado de México, específicamente en el grupo de trabajadores de vigilancia que está conformado por personas en un rango de edades entre 45 y 65 años, los cuales tienen turnos de 24 por 24 horas. es decir, 24 horas de trabajo por 24 horas de descanso. Su trabajo consiste en resguardar las instalaciones, equipo, mobiliario, laboratorios, abrir y cerrar oficinas, y además recibir a los estudiantes al ingresar verificando que no introduzcan objetos peligrosos o no permitidos revisando las mochilas uno por uno.

También realizan recorridos constantemente por todas las áreas de la institución, sin importar las condiciones climatológicas. En sus recesos no cuentan con áreas asignadas especialmente para el consumo de alimentos o descanso, solo cuentan con las cabinas de entrada y salida para resguardarse de las inclemencias del tiempo, además de ser muy pequeñas donde solo pueden estar dos personas como máximo. La institución está ubicada en una zona recién urbanizada y es muy solitaria, por lo que ha sido propicia para la delincuencia; se han presentado asaltos a maestros y estudiantes y en ocasiones los vigilantes han tenido que intervenir exponiendo su vida, debido a que no todos cuentan con armas de cargo y solo pueden acceder a toletes o gas pimienta. Lo anterior ha desencadenado una serie de afectaciones en su salud, tales como hipertensión, diabetes, problemas articulares, entre otras; dando lugar a incapacidades y ausentismo. La empresa que los contrata es externa y no considera las condiciones en que están desempeñando sus funciones, tales como factores psicosociales y condiciones ambientales.

Las condiciones de seguridad y salud en el trabajo difieren enormemente entre países, sectores económicos y grupos sociales, inclusive entre tipos de trabajo y profesiones, aun cuando en el caso de México la Secretaría del Trabajo y Previsión Social (STPS), establece los mecanismos (Leyes, Reglamentos, Normas, entre otras), mediante los cuales se rigen las relaciones Laborales y de Seguridad y Salud así como el mantenerlos actualizados según las propias necesidades de la sociedad y vigilar el cumplimiento de los mismos; y están muy enfocados en la industria manufacturera por la importancia de las certificaciones necesarias para la exportación de productos. Pero en el caso de servicios, aún están muy olvidados algunos sectores y grupos vulnerables tales como el del presente estudio.

Cabe reconocer que legalmente las instituciones gubernamentales están trabajando en mejorar las condiciones de los trabajadores, entre las acciones más trascendentes se encuentran las reformas publicadas el día 13 de noviembre de 2014, en el Diario Oficial de la Federación (DOF, 2014), referentes al Reglamento Federal de Seguridad y Salud en el Trabajo, entrando en vigor el 13 de febrero de 2015. Con el cual se abrogó el Reglamento Federal de Seguridad, Higiene y Medio Ambiente de Trabajo del 21 de enero de 1997. Con el objetivo de establecer las disposiciones en materia de Seguridad y Salud en el Trabajo que deberán observarse en los Centros, a efecto de contar con las condiciones que permitan prevenir riesgos y de esa manera, garantizar 
a los trabajadores el derecho a desempeñar sus actividades en entornos que seguro para su vida y su salud, con base en lo que señala la Ley Federal del Trabajo.

Lo anterior generó la entrada en vigor de otras Normas Oficiales Mexicanas como la NOM-035-1-2018STPS referente a factores psicosociales y la NOM-036-2018-STPS que trata sobre movimientos repetitivos y los factores de riesgo ergonómico pueden conllevar sobre esfuerzo físico, movimientos o posturas forzadas en el desempeño de las actividades laborales, con la consecuente fatiga, errores, accidentes y enfermedades profesionales derivados del diseño de las instalaciones, maquinaria, equipo, herramientas o puesto de trabajo.

"El término ergonomía proviene de las palabras griegas ergon (trabajo) y nomos (ley, norma o doctrina); la primera referencia a la ergonomía aparece en el libro Compendio de Ergonomía [2]. La International Ergonomics Association (IEA, 2016), define la ergonomía como la disciplina científica que se ocupa de la comprensión de la interacción entre los seres humanos y los demás elementos de un sistema, entre los objetivos de la ergonomía está contemplado el logro de la satisfacción en el trabajo, considerando las responsabilidades, actitudes, creencias y valores para el desarrollo personal, así como las diferencias individuales y culturales. Otro enfoque es el que considera la ergonomía como un "elemento educativo del entorno, que facilita la interactuación entre el individuo y su ambiente laboral; se traduce en mejor calidad de vida, reducción de posibles accidentes y enfermedades, incremento del bienestar, productividad, cuidado de la salud y medio ambiente" [3].

Considerando que para el desempeño de sus funciones los trabajadores de seguridad y vigilancia se ven sometidos a gran presión psicosocial y exigencia física, y con base en la NOM-035-STPS, en el presente estudio se analiza cómo se relaciona la ergonomía y el desempeño, en un sentido integral, en el que se incluyen aparatos, herramientas, materiales, métodos, con la finalidad de conocer la situación laboral que prevalece en los trabajadores y se enfoca principalmente a la productividad del desempeño, factores psicosociales y factores Ergonómicos, considerando que ésta disciplina está poco explotada en México y más aún, donde la función de un vigilante está expuesta a riesgos en su ámbito laboral, y son susceptibles a optimizar los recursos y condiciones laborables a través de una cultura ergonómica y en especial su vinculación con la administración.

Cabe destacar de acuerdo con Llaneza, que las quejas y demandas formuladas por los trabajadores referentes a las condiciones del ambiente físico, esconden problemas de origen psicosocial como la desmotivación, lo cual se refleja en la baja productividad y accidentabilidad en las empresas tanto manufactureras como de servicio [4].

La función de la Ergonomía en el diseño de lugares de trabajos y procesos cognitivos es controlar el impacto del entorno en el usuario y se basa en el principio de preservar la vida y la salud de las personas por encima de todo interés material y/o económico. Para lograrlo se establece adaptar el lugar del trabajo al trabajador y no a la inversa. Crear condiciones confortables, gratas y sobre todo seguras, así como un ambiente de cordialidad, que brinde al trabajador un sentido de pertenencia, aprecio por su trabajo y cuidado de su persona. Las condiciones adecuadas en la estación de trabajo que facilitan la realización de actividades, sin exponer la salud del trabajador, minimizan el ausentismo por enfermedad o por desmotivación, dando continuidad al flujo de procesos con un mejor desempeño e incremento de la productividad.

\section{DeSARRollo}

Los factores de riesgo psicosocial son aquellos que pueden provocar trastornos de ansiedad, no orgánicos del ciclo sueño-vigilia y de estrés grave y de adaptación, derivado de la naturaleza de las funciones del puesto de trabajo, el tipo de jornada de trabajo y la exposición a acontecimientos traumáticos severos o a actos de violencia laboral al trabajador, por el trabajo desarrollado. [5]

En el estudio se analizan factores relacionados con el requerimiento de la NOM-035-STPS, que fue dada a conocer para consulta pública el 26 de octubre de 2016 y fue publicada el 23 de octubre del 2018, para entrar en vigor el 23 de octubre de 2019. Cabe señalar que, algunos requisitos $(5.2,5.3,5.6,5.8,8.3,8.4,8.5$, y Capítulo 7) serán de cumplimiento obligatorio hasta el 23 de octubre de 2020. [6].

En ella se establecen los elementos para identificar, analizar y prevenir los factores de riesgo psicosocial, así como para promover un entorno organizacional favorable en los centros de trabajo e incrementar la Productividad en el desempeño administrativo. 
Para analizar las variables establecidas, se llevó a cabo el método Estadístico de Análisis de regresión para verificar la incidencia entre la variable productividad en el desempeño administrativo (Variable suma de productos ya que en forma conceptual se expresa como la producción de recursos por unidad de tiempo), y las Variables de la Ergonomía Organizacional; para darle sustento explicativo a las variables, se utiliza una matriz de correlaciones entre las variables y los Método Ergonómicos Rula (Rapid Upper Limb Assessment) para la evaluación ergonómica de la carga postural en las extremidades superiores y OWAS (Ovako Working Analysis System), su objetivo consiste en una evaluación del riesgo de carga postural en términos de frecuencia por gravedad [7].

En los últimos años, debido a la violencia que se ha desatado en el país, el personal de seguridad está sometido con mayor frecuencia al distrés laboral, que se conoce como el estrés desagradable, de acuerdo a la asociación española de seguridad ocupacional Prevencionar (2018), es un estrés que ocasiona un exceso de esfuerzo en relación a la carga de trabajo y va acompañado siempre de un desorden fisiológico, aceleración de las funciones, hiperactividad, acortamiento muscular, somatizaciones, en suma: envejecimiento prematuro, mismos que se ha asociado con el incremento de las exigencias laborales que tienen que ser asumidas con la finalidad de mejorar el salario y en la participación en diversos programas por estímulos económicos. [8]

Existen referencias anteriores de estudios similares sobre las presiones a las cuales se ven sometidos los trabajadores de la Universidad Autónoma Metropolitana, aun cuando fue aplicado a profesores y administrativos, refleja que un trabajo de servicios no es tan relajado como aparenta; en dichos estudios se recopiló la cuantificación porcentual de los daños encontrando los siguientes resultados: la migraña fue de $23 \%$, la disfonía con $38 \%$, un tercio de su población se sentía fatigado todo el tiempo; la fatiga neurovisual $25 \%$, cefalea tensional de $24 \%$, problemas para conciliar el sueño $32 \%$, trastornos músculo-esqueléticos $38 \%$ y los lumbares $26 \%$. El estrés laboral también es generado por permanecer más tiempo de la jornada laboral en el mismo lugar y éste trastorno también se presentó en la población UNAM, asociado al hecho de recibir órdenes confusas y poco claras por parte del jefe [9].

También es muy importante considerar el costo beneficio de las aplicaciones ergonómicas y su incidencia en la productividad para incentivar las aplicaciones Ergonómicas se debe justificar su rentabilidad. Para ello existen diversos métodos, y uno de ellos es el método Occupational Repetitive Action (OCRA), método de evaluación del riesgo de movimientos repetitivos en extremidades superiores. Los niveles de riesgo permiten una ponderación y una de las ventajas es que se pueden identificar el porcentaje de trabajadores con riesgo de adquirir daños acumulativos y que potencialmente, van disminuyendo su productividad de forma paulatina y adquiriendo así mismo una enfermedad profesional.

Otro factor relevante es que la matrícula estudiantil incrementa sin que la planta laboral crezca en la misma proporción y en ocasiones tiende a disminuir. Este conjunto de hechos racionaliza recursos económicos e instrumentales de los trabajadores, aumentando la carga con la consecuencia de afectar su productividad con riesgo de dañar también su salud física y mental. Para su análisis se dividió en Ergonomía Ambiental, Ergonomía Física y Ergonomía del Factor Humano, los cuales se explican a continuación:

- Ergonomía ambiental: Es la rama de la Ergonomía, especializada en el estudio de los factores ambientales, generalmente físicos, que constituyen el entorno del sistema formado por la persona y el equipo de trabajo y su influencia en los aspectos relacionados con la seguridad, la eficiencia y la confortabilidad. Incluye el estudio de los ambientes térmico, visual, acústico, mecánico, electromagnético y de distribución del puesto de trabajo [10].

- Ergonomía Física: Se preocupa de las características anatómicas, antropométricas, fisiológicas y biomecánicas del usuario, en tanto que se relacionan con la actividad física [11].

- Ergonomía del Factor Humano: Es la disciplina científica relacionada con el conocimiento de la interacción entre el ser humano y otros elementos de un sistema, y la profesión que aplica la teoría, principios, datos y métodos para diseñar buscando optimizar el bienestar humano y la ejecución del Sistema Global. Condiciones que se encuentran presentes en una situación laboral y que están directamente relacionadas con la organización, el contenido del trabajo y la realización de la tarea, y 
que tienen capacidad para afectar tanto al bienestar o a la salud (física, psíquica o social) del trabajador como al desarrollo del trabajo [6].

Debido a que en la investigación se evalúan, condiciones ambientales y puestos de trabajo, la muestra se enfocó a trabajadores de seguridad y vigilancia, por contar con las características requeridas; sin embargo, no se incluyó a todos; la investigación se vio limitada por los tiempos de trabajo del personal. Se identificaron los factores de Ergonomía Organizacional, que a continuación se presentan:

Ergonomía del Factor humano.

$$
\begin{array}{ll}
\text { - } & \text { Sexo } \\
\text { - } & \text { Edad } \\
\text { - } & \text { Participación, implicación, responsabilidad } \\
\text { - } & \text { Formación, comunicación e Información } \\
\text { - } & \text { Gestión de tiempo } \\
\text { - } & \text { Cohesión de grupo }
\end{array}
$$

Ergonomía Ambiental.

- Ambiente luminoso

- Ambiente térmico

- Ambiente sonoro

Ergonomía Física.

- Postura estática

- Postura dinámica

- Repeticiones

- Carga mental

- Gasto metabólico

- Antropometría

\section{A. Método}

La muestra se conformó de 31 trabajadores, en representación del 41\% de la población total, como base para estudios posteriores; fueron encuestados todos los participantes de la muestra seleccionada, con el propósito de obtener información confiable, en los parámetros estadísticos de la Productividad del desempeño, en relación con la Ergonomía Organizacional. Un instrumento de medición, según [1] es, en principio, cualquier recurso de que se vale el investigador para acercarse a los fenómenos y extraer de ellos información. De este modo, el instrumento sintetiza en sí toda la labor previa de investigación: resume los aportes del marco teórico al seleccionar datos que corresponden a los indicadores y, por lo tanto, a las variables o conceptos utilizados.

\section{1) Instrumentos de evaluación}

El Instrumento de medición referente a la evaluación de Ergonomía Organizacional fue adaptado por la autora con base en el Manual para la evaluación y prevención de riesgos ergonómicos y psicosociales en la PYME del Instituto Nacional de Seguridad e Higiene en el Trabajo (INSHT, 2014), del Ministerio del Trabajo y asuntos sociales, específicamente en las listas de comprobación básica de la colección Cuestionarios; y en la Normatividad vigente en México de la STPS (2014), referentes al Ruido (NOM-011-STPS-2011), Temperaturas (NOM-015-STPS-2011) y Luminiscencia (NOM-025-STPS-2001).

También se aplica para la Ergonomía del Factor Humano el Instrumento de Factores Psicosociales, identificación de situaciones de riesgo, del Instituto Navarro de Salud Laboral, el cual se adaptó para la aplicación de la NOM-035-1-2018-STPS; cabe hacer mención que la Norma establece dos tipos de 
cuestionarios: uno para trabajadores con vivencia de hechos traumáticos y el segundo sin hechos traumáticos, en la presente investigación por ser el estudio inicial se aplicó sin eventos traumáticos y en futuros estudios se abordarán ambos cuestionarios. En el Instrumento se identifican cuatro variables relacionadas con el entorno laboral y que afectan a la salud del trabajador y al desarrollo de la tarea a realizar. Consta de 30 preguntas las cuales se muestran en el Anexo 1 [12].

Estas variables son:

- Participación, Implicación, Responsabilidad.

- Formación, Información, Comunicación.

- Gestión del tiempo.

- Cohesión de grupo.

Este método de evaluación tiene como objetivo obtener información, respecto a las cuatro variables anteriores, que permita detectar situaciones en la organización desfavorables o muy insatisfactorias, que pueden ser fuente de riesgo para la salud de los trabajadores. Con el análisis de los resultados obtenidos se adquiere una visión general de la empresa respecto a los factores de riesgo psicosocial. [13]

Se pretende que esto sea el punto de partida para una evaluación específica más concreta en aquellas áreas en las que aparezcan aspectos deficitarios. Además de los cuatro factores mencionados anteriormente, se han incluido tres preguntas con el objetivo de reconocer la vulnerabilidad a la existencia de hostigamiento psicológico en el trabajo. La metodología se basa en la aplicación de una serie de cuestionarios en la empresa a una muestra representativa de la plantilla (se recomienda una muestra en torno al 20-30\% del total), condición que se cumple en la presente investigación.

Los resultados obtenidos en cada cuestionario se trasladan a una hoja de valoración y de categorización que permite diagnosticar. El cuestionario lo componen 30 preguntas, con varias alternativas de respuesta y una opción de respuesta cualitativa (Observaciones) que posibilita una aclaración de la respuesta aportada y se han agrupado en cuatro variables con sus respectivos factores: [10]

\section{a) Participación, Implicación, Responsabilidad}

Especifica el grado de libertad e independencia que tiene el trabajador para controlar y organizar su propio trabajo y para determinar los métodos a utilizar, teniendo en cuenta siempre los principios preventivos. Define el grado de autonomía del trabajador para tomar decisiones. Se entiende que un trabajo saludable debe ofrecer a las personas la posibilidad de tomar decisiones.

En la dimensión Participación, Implicación, Responsabilidad se han integrado estos factores:

- Autonomía

- Trabajo en equipo

- Iniciativa

- Control sobre la tarea

- Control sobre el trabajador

- Rotación

- Supervisión

- Enriquecimiento de tareas

Las preguntas del cuestionario que se refieren a esta variable son: 1, 2, 8, 9, 13, 18, 19, 20 y 25.

\section{b) Formación, Información, Comunicación}

Se refiere al grado de interés personal que la organización demuestra por los trabajadores, facilitando el flujo de información es necesarias para el correcto desarrollo de las tareas. Las funciones y/o atribuciones de cada persona, dentro de la organización, tienen que estar bien definidas para garantizar la adaptación óptima entre 
los puestos de trabajo y las personas que los ocupan. En el área de Formación, Información, Comunicación se han incorporado los siguientes aspectos:

- Flujos de comunicación

- Acogida

- Adecuación persona-trabajo

- Reconocimiento

- $\quad$ Adiestramiento

- Descripción de puesto de trabajo

- Aislamiento

Las preguntas del cuestionario que se refieren a esta variable son: 4, 5, 11, 16, 17, 24 y 26.

\section{c) Gestión de tiempo}

Establece el nivel de autonomía concedida al trabajador para determinar la cadencia y ritmo de su trabajo, la distribución de las pausas y la elección de las vacaciones de acuerdo con sus necesidades personales. En la dimensión Gestión del Tiempo se han integrado estos factores:

- Ritmo de trabajo

- Apremio de tiempo

- $\quad$ Carga de trabajo

- Autonomía temporal

- Fatiga

Las preguntas del cuestionario que se refieren a esta variable son: 3, 10, 14, 15 y 22.

\section{d) Cohesión de grupo}

Definimos cohesión como el patrón de estructura del grupo, de las relaciones que emergen entre los miembros del grupo. Este concepto incluye aspectos como solidaridad, atracción, ética, clima o sentido de comunidad. La influencia de la cohesión en el grupo se manifiesta en una mayor o menor participación de sus miembros y en la conformidad hacia la mayoría.

La variable Cohesión de grupo contiene los siguientes aspectos:

- Clima social

- Manejo de conflictos

- Cooperación

- Ambiente de trabajo

Las preguntas del cuestionario que se refieren a esta variable son: 6, 7, 12, 21, 23 y 27.

\section{2) Hostigamiento psicológico (mobbing)}

El hostigamiento psicológico en el trabajo hace referencia a aquellas situaciones en las que una persona o un grupo de personas ejerce un conjunto de comportamientos caracterizados por una violencia psicológica extrema, de forma sistemática y durante un tiempo prolongado, sobre otra persona en el lugar de trabajo.

El efecto que se pretende alcanzar es el de intimidar, apocar, reducir y consumir emocional e intelectualmente a la víctima, con vistas a eliminarla de la organización. Las preguntas del cuestionario que se refieren a esta variable son: 28,29 y 30 .

Importante: En el supuesto de obtener un punto en alguna de estas tres preguntas se debe profundizar con un cuestionario específico sobre identificación de hostigamiento psicológico en el trabajo con el fín de descartar la posibilidad de que se esté dando esta situación, por sus nefastas consecuencias. [10] 


\section{Recursos}

Equipo de medición de Condiciones ambientales.

- $\quad$ Unidad Central de Procesamiento: Science cube Pro.

- $\quad$ Sensor de temperatura.

- $\quad$ Sensor de luz.

- $\quad$ Sensor de ruido.

\section{RECOLECCIÓN DE DATOS}

Se analizaron cada uno de los métodos de evaluación ergonómica para identificar cuál era el más apropiado para esta investigación, optando por elegir la lista de revisión (check list) ya que es un instrumento práctico, rápido y fácil de utilizar para revisar las condiciones de riesgo ergonómico a los que se somete un usuario al desarrollar una actividad, proporcionan la información preliminar que permite identificar las principales áreas o condiciones de riesgo a evaluar con mayor detalle, repeticiones, posturas, condiciones, entre otros factores. Para la aplicación se llevó a cabo una visita a cada uno de los puestos a estudiar, para conocer el proceso de trabajo, las tareas que se llevan a cabo en la organización y características de trabajo se seleccionaron a personas puestos y momentos de la jornada.

Previamente, fue necesario establecer claramente con qué criterios se realizaría esta selección, por lo tanto se recabó información referente al tipo de plaza, cantidad de horas laborables y tipo de personal para poder facilitar la selección de la muestra, para lo cual se realizaron una serie de observaciones y entrevistas generales acerca de los diversos aspectos que son base del procedimiento a seguir., así mismo se aplicó la lista de comprobación básica del sitio de trabajo modificada del Check List emitido por la Occupational Safety and Health Administration (OSHA, 2003), debido a la diversidad de horarios y funciones del personal del área de estudio, una vez seleccionado los criterios se pudo aplicar al trabajo de investigación a 35 trabajadores de la siguiente manera:

El Test de Autoevaluación de Puestos de Trabajo, en forma de entrevista para responder las dudas que fueran saliendo durante la aplicación.

La evaluación del ambiente luminoso se realizaron mediciones en los puestos de trabajo seleccionados, utilizando como equipo un medidor de luminancia, el cual proporciona datos de iluminación en luxes, con la siguiente escala:

- Inadecuada

- Insuficiente

- $\quad$ Suficiente

- Adecuada

- Muy adecuada

\section{IV.RESULTADOS}

En el aspecto de la vibración es de $4.3 \mathrm{HTZ}$, y con base a la NORMA-024-STPS-2001 [8], se muestra la Tabla 1 y el trabajador se encuentra laborando en un estado óptimo lo cual indica que está en el rango aceptable.

El valor de la iluminación es de 158.92 lux, se realiza a la intemperie, permaneciendo alrededor de 5 horas al día y con algunos lapsos de descansos en algún lugar sombreado y de acuerdo con lo establecido en la norma el rango aceptable para realizar su trabajo.

La condición a la que está trabajando es de $99 \mathrm{~dB}$ durante 4 horas, y de acuerdo con la norma como se observa en la tabla nos indica que están sobrepasando el límite permisible de exposición. 
TABLA 1

CONDiCIONES AMBIENTALES

Medidas tomadas a medio día

\begin{tabular}{lclc}
\hline Ruido & $64 \mathrm{~dB}$ & Ruido & $109.89 \mathrm{~dB}$ \\
Humedad Relativa & $97.2 \mathrm{HR}$ & Humedad Relativa & $62.7 \mathrm{HR}$ \\
\hline Iluminación & $107.52 \mathrm{Lux}$ & Iluminación & $158.92 \mathrm{Lux}$ \\
Temperatura & $17.90{ }^{\circ} \mathrm{C}$ & Temperatura & $22.50{ }^{\circ} \mathrm{C}$ \\
Vibración & $4.3 \mathrm{~Hz}$ & Vibración & $4.4 \mathrm{~Hz}$ \\
Frecuencia cardiaca & $101.21 \mathrm{ps}$ & Frecuencia cardiaca & $108.9 \mathrm{ps}$ \\
\hline
\end{tabular}

La validación y correlación se muestran en las pruebas de normalidad obtenidas como se muestra en la Tabla 2.

TABLA 2

PRUEBAS DE NORMALIDAD

\begin{tabular}{ccccccc}
\hline & \multicolumn{3}{c}{ Kolmogorov-Smirnov $^{\text {a }}$} & \multicolumn{3}{c}{ Shapiro-Wilk } \\
& Estadístico & gl & Sig. & Estadístico & gl & Sig. \\
\hline Productividad & .059 & 246 & .095 & .993 & 246 & .350 \\
$\begin{array}{c}\text { Ergonomía } \\
\text { Organizacional }\end{array}$ & .054 & 246 & .078 & .993 & 246 & .268 \\
\hline
\end{tabular}

La estimación de correlación lineal entre la Ergonomía y la Productividad tiene un coeficiente de determinación alto con un nivel del $85.5 \%$, como se observa en el análisis de la Tabla 3.

TABLA 3

RESUMEN DEL MODELO Y ESTIMACIONES DE LOS PARÁMETROS

\begin{tabular}{lccccccc}
\hline & & & & & & \multicolumn{3}{c}{$\begin{array}{c}\text { Estimaciones de los } \\
\text { parámetros }\end{array}$} \\
\hline & & Resumen del modelo & & & & \\
Ecuación & cuadrado & $\mathrm{F}$ & gl1 & g12 & Sig. & Constante & b1 \\
Lineal &, 855 & 15,441 & 1 & 28 &, 001 &, 105 & 1,984 \\
\hline
\end{tabular}

Variable dependiente: Productividad | Variable independiente Ergonomía

Con los datos obtenidos en la correlación y el modelo de estimación de los parámetros se llevó a cabo la interpolación de las variables para identificar la tendencia entre las variables (véase Fig. 1). 


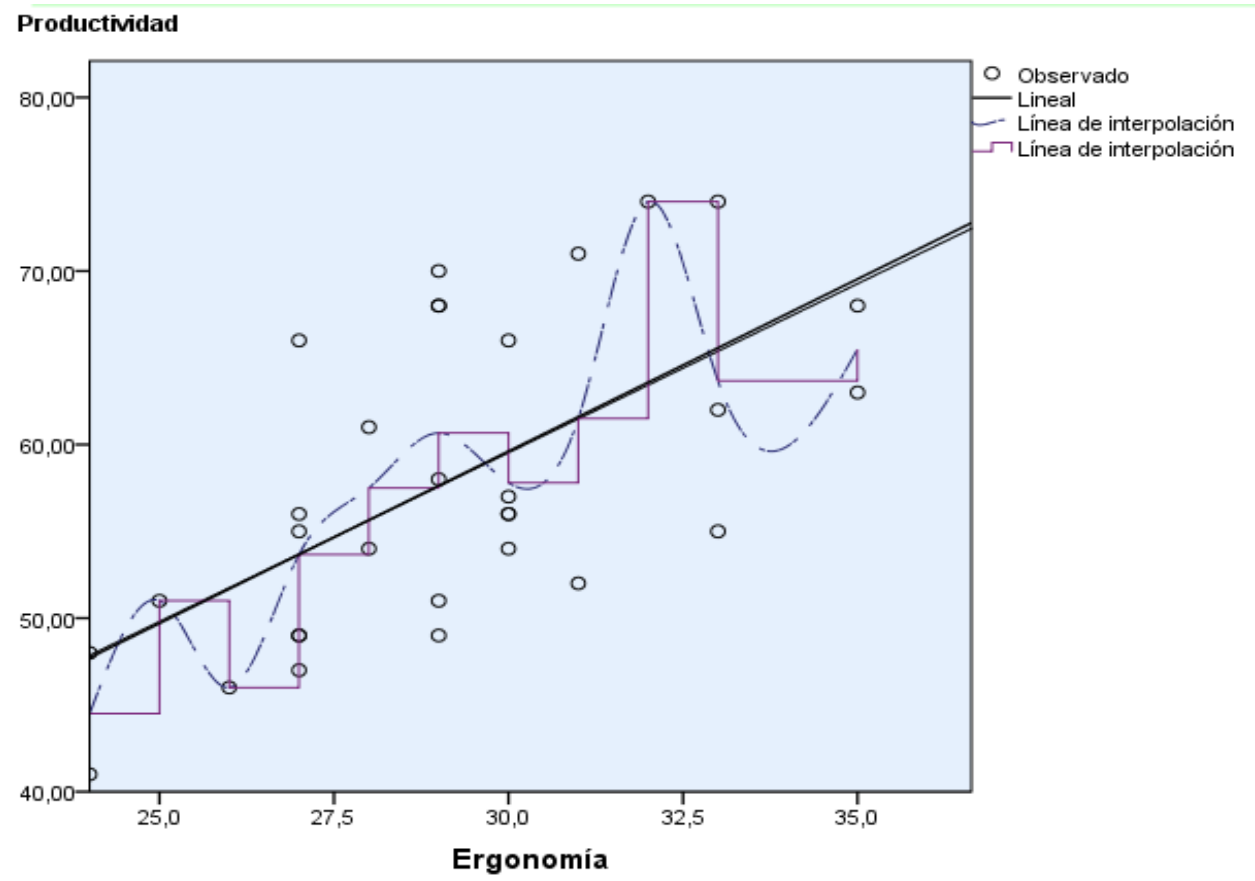

Fig. 1. Análisis de relación entre Ergonomía y Productividad.

En la institución la mayoría de los elementos de seguridad y vigilancia predomina el género masculino (véase Fig. 2).

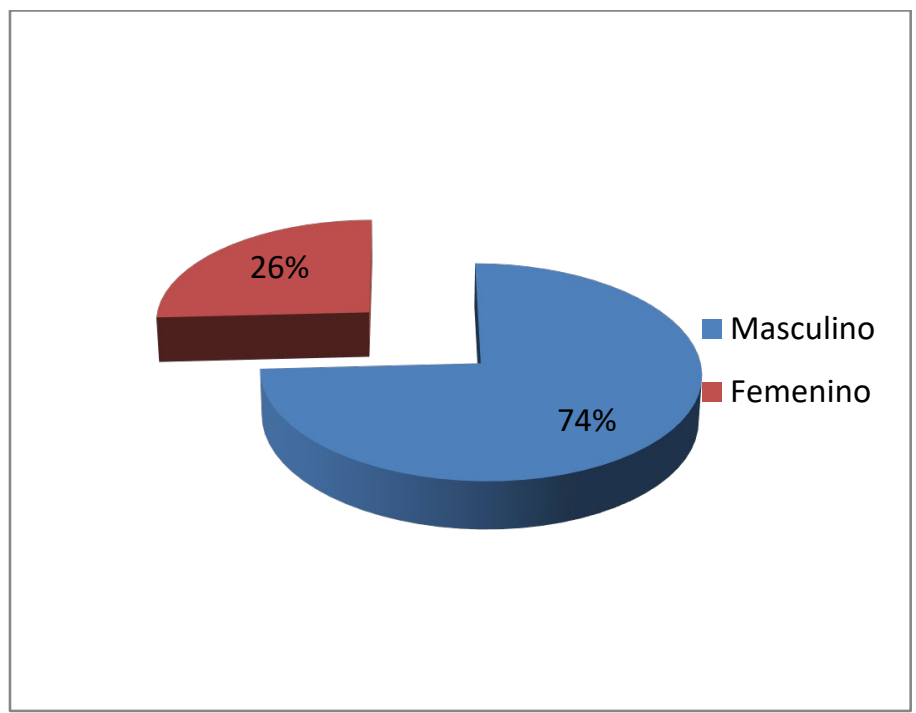

Fig. 2. Distribución por género. 
Destaca que las edades de los trabajadores de seguridad y vigilancia por encima de los 50 años (véase Fig. 3).

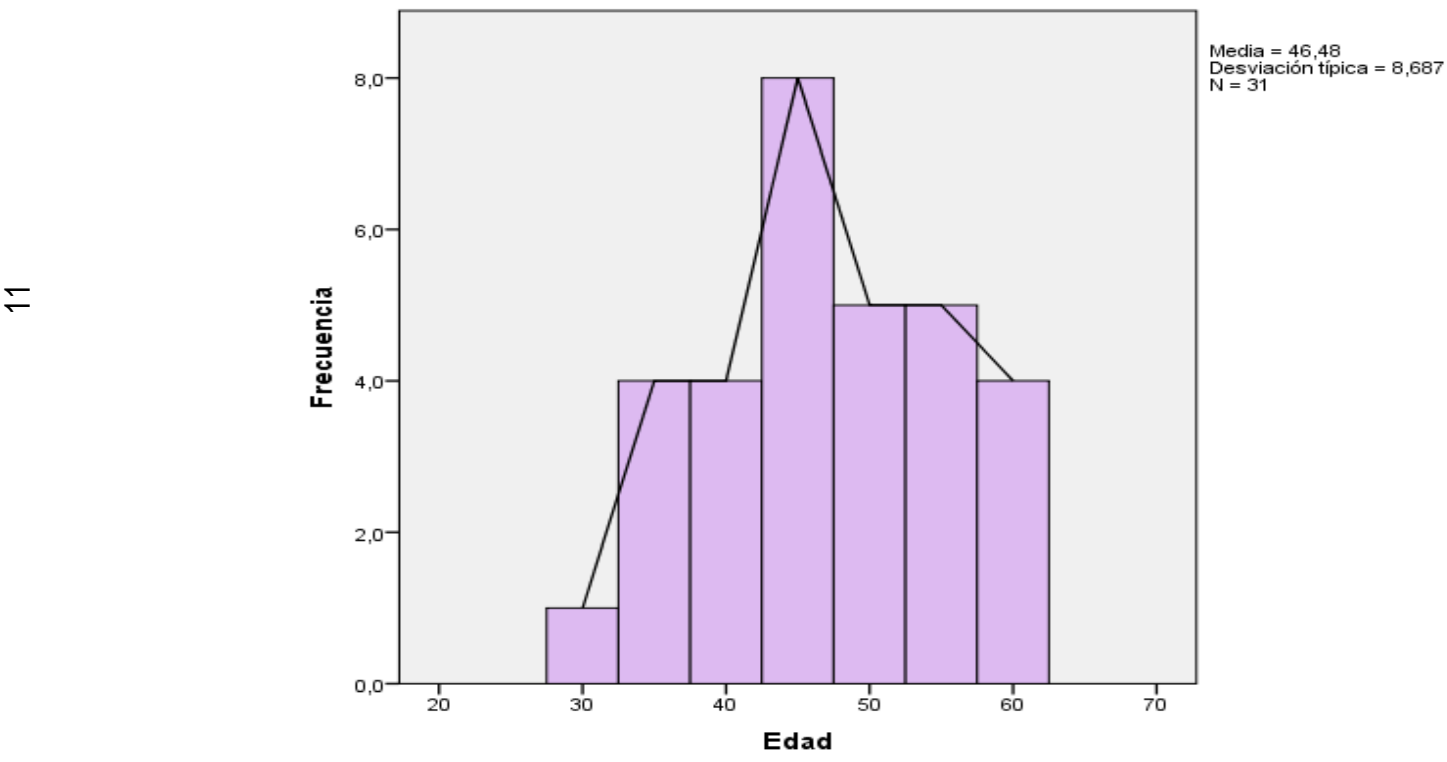

Fig. 3. Histograma de tendencia por edades.

Para llevar a cabo la evaluación de los movimientos en el método RULA se utilizó el formato diseñado por Ergonomics Plus Inc. [14] (véase Fig. 4).

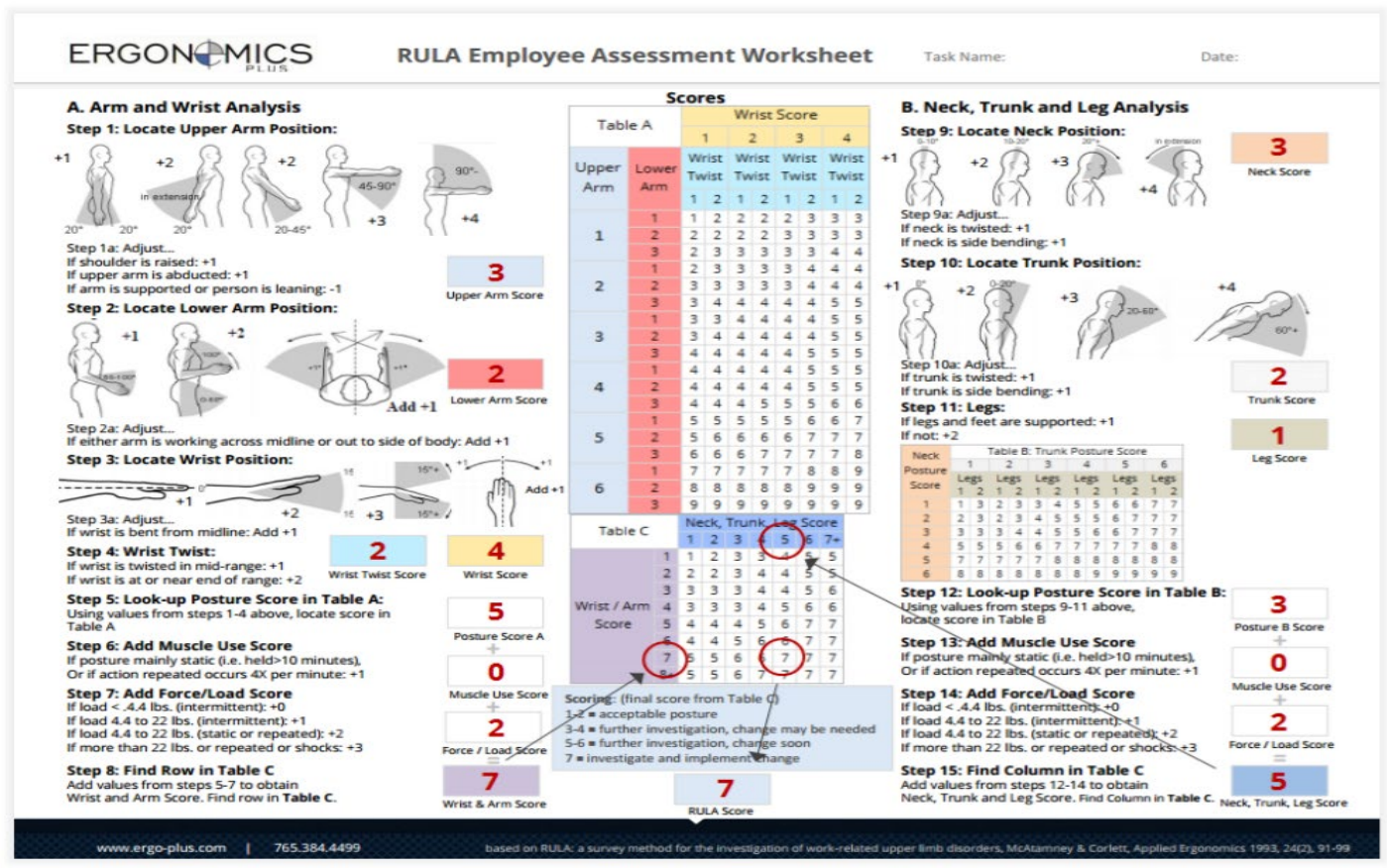

Final RULA Score $=7$

Fig. 4. Resultados Método RULA.

Fuente: Formato de Ergonomics Plus Inc. [14]. 
Los resultados del cuestionario en cada uno de los criterios mostraron la necesidad de tomar medidas para un mejor clima laboral:

$\checkmark$ Participación, Implicación, Responsabilidad: Adecuada

$\checkmark$ Formación, Información, Comunicación: Deficiente

$\checkmark$ Gestión del Tiempo: Insuficiente

$\checkmark$ Cohesión de Grupo: Inadecuada

\section{CONCLUSIONES}

Se encontró un alta correlación entre los factores de Ergonomía Organizacional y los efectos en la salud física, mental y emocional de los trabajadores, lo cual implica la gran necesidad de considerar cambios en las unidades y centro de trabajo rediseñando, acondicionando y evaluando conforme a la normatividad vigente de la Secretaría del Trabajo y Previsión Social, se identificaron riesgos psicosociales internos y externos en que se desempeñan los trabajadores; además se requiere un estudio más exhaustivo por puesto de trabajo y a todos los niveles de la organización para que los cambios sean integrales y así evitar ambientes nocivos tanto físicos, como organizacionales y humanos.

Con base en la evaluación a través del Método RULA se analizaron las extremidades superiores y con el método OWAS se analizaron brazos, tronco, piernas, cabeza la puntuación obtenida es la más alta en ambos métodos, lo que implica que el trabajador presente fatiga muscular por posturas forzadas, es decir, que una o varias regiones anatómicas dejan de estar en una posición natural de confort y pasan a una posición (forzada) que genera hiperextensiones, hiperflexiones o hiperrotaciones, osteoarticulares con la consecuente producción de lesiones por sobrecarga y Disfunciones Traumático Acumulativas (DTA); existen numerosas actividades en las que el trabajador de seguridad puede adoptar posturas forzadas, siendo comunes bipedestación o sedestación prolongadas [9].

En la ergonomía física, las condiciones ambientales en lo referente al ruido sobrepasan los límites aceptables establecidos por la Norma Oficial Mexicana NOM-011-STPS-2001, ocasionando que los trabajadores estén expuestos a stress, irritabilidad, hipertensión arterial y puede estar asociado a otras situaciones de riesgo como cardiopatías.

La zona donde se ubica la institución, como ya se mencionó, es aislada y con poca afluencia por lo cual es vulnerable a delincuencia y vandalismo. Es muy importante enfatizar que la siniestralidad causa grandes efectos de stress, irritabilidad, hipertensión arterial y temor al desempeñar sus funciones cotidianas, además no se han establecido oficialmente protocolos de seguridad y el equipo de protección es escaso o nulo, dependiendo la antigüedad del trabajador; tales como armas de cargo, chalecos salvavidas, escudos ante manifestaciones estudiantiles y todo lo relacionado con equipo de protección personal, esto genera una gran tensión y sentimiento de vulnerabilidad como lo han expresado, y somatizado en malestares y deterioro de su salud. [13].

Con lo que se puede concluir que la suma de riesgo por factores psicosociales según lo contemplado en la NOM-035-1-2018-STPS, confirma la existencia de alto riesgo en el desempeño de la actividad y su incidencia en el desempeño y productividad. Resulta importante que se consideren los aspectos mencionados debido a que la salud de los trabajadores se encuentra expuesta con diversos factores, es necesario una intervención ergonómica para evitar riesgo de adquirir, enfermedades, lesiones y accidentes en las áreas de trabajo, además de proporcionar seguridad y confort al trabajador, lo cual se verá reflejado en mayor fidelización y mejor desempeño laboral.

\section{REFERENCIAS}

[1] E. Apud, F. Meyer, "La importancia de la ergonomía para los profesionales de la salud," Ciencia y enfermería, vol. 9, no. 1, pp. 15-20, 2003, doi: http://dx.doi.org/10.4067/S0717-95532003000100003

[2] J. L. González, Lesiones musculo-esqueléticas, 2007, available: http://www.ergocupacional.com/4910/57873.html

[3] Universidad Politécnica de Valencia, Métodos de evaluación de la ergonomía de puestos de trabajo, Ergonautas, 2006, available: https://www.ergonautas.upv.es/metodos-evaluacion-ergonomica.html 
[4] J. Llaneza-Álvarez, Ergonomía y psicosociología aplicada. Manual para la formación del especialista, 10ª ed., Valladolid, LEX NOVA, España, 2008.

[5] B. Moreno, "Factores y riesgos laborales psicosociales: conceptualización, historia y cambios actuales," Medicina y Seguridad del Trabajo, vol. 57, no.1, pp. 4-19, 2011, doi: http://dx.doi.org/10.4321/S0465-546X2011000500002

[6] Diario Oficial de la Federación, 30 diciembre 2019, available: https://www.dof.gob.mx/nota_detalle.php?codigo=5541828\&fecha=23/10/2018

[7] J. A. Diego-Mas, Evaluación Postural Mediante El Método OWAS, Ergonautas, Universidad Politécnica de Valencia, 2015, available: https://www.ergonautas.upv.es/metodos/owas/owas-ayuda.php

[8] M. A. Carranza, Secretaría del Trabajo y Prevención Social, 14 junio 2019, available: http://www.stps.gob.mx/bp/secciones/dgsst/normatividad/normas/Nom-015.pdf

[9] M. A. Carranza, Diario Oficial de la Federación, 01 noviembre 2002, available: http://dof.gob.mx/nota_detalle.php?codigo=737289\&fecha=11/01/2002

[10] D. Prado, La fatiga física y su recuperación, 2019, available: http://www.imf-formacion.com/blog/prevencion-riesgoslaborales/actualidad-laboral/la-fatiga-fisica-y-su-recuperacion/

[11] Instituto Nacional de Seguros, Principios de Ergonomía, 2012, available: https://www.inscr.com/media/2631/1007800_principiosdeergonomc3ada_web.pdf

[12] M. Lahera, J. J. Góngora, Factores Psicosociales. Identificación de situaciones de riesgo, INSL, Navarra, España, 2014.

[13] L. Mertens, M. Falcón, Salud y seguridad en el trabajo y el papel de la formación en México, CEPAL, Santiago de Chile, 2004.

[14] Ergonomic Plus Inc, RULA: A Step-by-Step Guide, 2019, Ergonomics Plus Inc., www.ergo-plus.com 


\section{ANEXO 1}

INSTRUMENTO DE FACTORES PSICOSOCIALES, IDENTIFICACIÓN DE SITUACIONES DE RIESGO, DEL INSTITUTO NAVARRO DE SALUd LABORAL (INSL)

\section{FACTORES PSICOSOCIALES}

Este cuestionario pretende obtener una visión global de su organización respecto a los factores de riesgo de tipo psicosocial.

A continuación le presentamos una serie de preguntas con varias alternativas de respuesta. Desde un punto de vista general, elija la opción que más se acerque a su empresa.

Le informamos de que cualquier dato que usted aporte en el cuestionario será tratado de manera CONFIDENCIAL.

En cada pregunta le ofrecemos un apartado denominado OBSERVACIONES, utilícelo siempre que desee hacer alguna aclaración o puntualización al respecto, cuando necesite explicar alguna de sus opciones o cuando la alternativa escogida no englobe a la totalidad de las personas que forman parte de su empresa.

Ante cualquier duda que le surja durante la ejecución de la encuesta, no dude en pedir cualquier aclaración o información adicional.

Muchas gracias por su colaboración.

DATOS RELATIVOS A LA EMPRESA

Actividad a la que se dedica:

Sector al que pertenece:

Jornada laboral 1 turno 2 turnos 3 turnos MIXTO 


\section{Pregunta 1}

¿Usted como trabajador de seguridad tiene libertad para decidir cómo hacer su propio trabajo?
A. No.
B. Sí, ocasionalmente.
C. Sí, cuando la tarea se lo permite.
D. Sí, es la práctica habitual.

\section{Pregunta 4}

¿Usted como trabajador de seguridad dispone de la información y de los medios necesarios (equipo, herramientas, etc.) para realizar su tarea?

A. No.

B. Sí, algunas veces.

C. Sí, habitualmente.

D. Sí, siempre.
A B C D

\section{Pregunta 2}

Existe un procedimiento de atención a las posibles sugerencias $y / o$ reclamaciones planteadas por los trabajadores?
A. No, no existe.
B. Sí, aunque en la práctica no se utiliza.
C. Sí, se utiliza ocasionalmente.
D. Sí, se utiliza habitualmente.

\section{A B C D}

\section{Pregunta 3}

¿Usted como trabajador de seguridad tiene la posibilidad de ejercer el control sobre su ritmo de trabajo?
A. No.
B. Sí, ocasionalmente.
C. Sí, cuando la tarea se lo permite.
D. Sí, es la práctica habitual.

$$
\text { A B C D }
$$

\section{A $B$ C D}

\section{Pregunta 5}

Ante la incorporación de nuevos trabajadores, ¿se les informa de los riesgos generales $y$ específicos del puesto?

A. No.

B. Sí, oralmente.

C. Sí, por escrito.

E. Sí, por escrito y o ralmente

\section{A $\quad$ B $\quad$ C $\quad$ D}

\section{Pregunta 6}

Cuando usted como trabajador de seguridad necesita ayuda y/o tiene cualquier duda acude a:

A. Un compañero de otro puesto.

B. Una persona asignada. (mantenimiento,

C. Un encargado y/o jefe superior.

D. No tiene esa opción por cualquier motivo.

$$
\text { A B C D }
$$

\section{Pregunta 7}

Las situaciones de conflictividad entre trabajadores, ¿se intentan solucionar de manera abierta y clara?

A. No.

B. Sí, por medio de la intervención del mando.

C. Sí, entre todos los afectados.

D. Sí, mediante otros procedimientos.

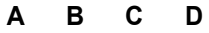

\section{Pregunta 8}

¿Pueden los trabajadores elegir sus días de vacaciones?

A. No, la empresa cierra por vacaciones en periodos fijos.

B. No, la empresa distribuye periodos vacacionales, sin tener en cuenta las necesidades de los trabajadores.

C. Sí, la empresa concede o no a demanda del trabajador.

D. Sí, los trabajadores se organizan entre ellos, teniendo en cuenta la continuidad de la actividad

\section{A $\quad$ B $\quad$ C $\quad$ D}

\section{Pregunta 9}

¿Usted como trabajador de seguridad interviene $y / 0$ corrige los incidentes en su puesto de trabajo (equipo, máquina, etc.)?

A. No, es función del mando superior o persona encargada.

B. Sí, sólo incidentes menores afectados.

C. Sí, cualquier incidente.

A B C 


\section{Pregunta 10}

¿Usted como trabajador de seguridad tiene posibilidad de realizar pausas dependiendo del esfuerzo (físico y/o mental) requerido

A. No, por la continuidad del proceso.

B. No, por otras causas.

C. Sí, las establecidas.

D. Sí, según necesidades.

\section{A $\quad$ B $\quad$ C $\quad$ D}

\section{Pregunta 11}

¿Se utilizan medios formales para transmitir informaciones $y$ comunicaciones a los trabajadores?

A. No

B. Charlas, asambleas.

C. Comunicados escritos Sí, medios orales y escritos

\section{A $\quad$ B $\quad$ C $\quad$ D}

\section{Pregunta 12}

En términos generales, ¿el ambiente de trabajo posibilita relaciones amistosas?
A. No.
B. Sí, a veces.
C. Sí, habitualmente
D. Sí, siempre

A B C D

Pregunta 13

La actuación del mando intermedio respecto a sus subordinados es:

A. Únicamente marca los objetivos individuales a alcanzar por el trabajador.

B. Colabora con Usted como trabajador de seguridad en la consecución de fines.

C. Fomenta la consecución de objetivos en equipo.

\section{A $\quad$ B $\quad$ C $\quad$ D}

\section{Pregunta 14}

¿Se recuperan los retrasos?
A. No.
B. Sí, durante las pausas.
C. Sí, incrementando el ritmo de trabajo.
D. Sí, alargando la jornada

\section{A $\quad B \quad C \quad D$}

\section{Pregunta 15}

\section{¿Cuál es el criterio de retribución} al trabajador?

A. Salario por hora (fijo).

B. Salario más prima colectiva.

C. Salario más prima individual.

\section{Pregunta 16}

¿Se facilitan las instrucciones precisas a los trabajadores sobre el modo correcto y seguro de realizar las tareas?

A. No.

B. Sí, de forma oral.

C. Sí, de forma escrita (instrucciones).

D. Sí, de forma oral y escrita.

A B C D

\section{Pregunta 17}

¿Usted como trabajador de seguridad tiene la posibilidad de hablar durante la realización de su tarea?

A. No, por la ubicación del trabajador.

B. No, por el ruido.

C. No, por otros motivos.

D. Sí, algunas palabras.

E. Sí, conversaciones más largas

\section{A $B$ C $\quad$ D $E$}

\section{Pregunta 18}

¿Han recibido los mandos intermedios formación para el desempeño de sus funciones?

A. No

B. Sí, aunque no ha habido cambios significativos en el estilo de mando.

C. Sí, algunos mandos han modificado sus estilos significativamente.

D. Sí, la mayoría ha modificado su estilo de mando.

A $\quad$ B $\quad$ C $\quad$ D 


\section{•egunta 19}

Existe la posibilidad de organizar el trabajo en equipo?

No.

Sí, cuando la tarea se lo permite. Sí, en función del tiempo disponible.

₹ . Sí, siempre se hace en equipo

\section{A $\quad$ B $\quad$ C $\quad$ D}

egunta 20

Jsted como trabajador de seguridad controla el resultado de su trabajo y puede corregir los errores cometidos o defectos?
A. No.
B. Sí, ocasionalmente.
C. Sí, habitualmente.
D. Sí, cualquier error.

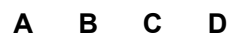

\section{'egunta 21}

S̀e organizan, de forma espontánea, eventos en los que participa la mayoría de la plantilla?

En términos generales, ¿e ambiente de trabajo posibilita relaciones No.

- Sí, una o dos veces al año

- Sí, varias veces al año, según surja el motivo.

\section{Pregunta 22}

¿Usted como trabajador de seguridad puede detener el trabajo o ausentarse de su puesto?

A. No, por el proceso productivo.

C. Sí, con un sustituto.

D. Sí, sin que nadie le sustituya.
B. No, por otros motivos.

\section{Pregunta 23}

¿Existe en general un buen clima laboral en su lugar de trabajo?

A. No.

B. Sí, a veces.

C. Sí, habitualmente.

D. Sí, siempre.

\section{Pregunta 25}

¿Usted como trabajador de seguridad tiene la opción de cambiar de puesto y/o de tarea a lo largo de su jornada laboral?

A. No.

B. Se cambia de manera excepcional.

C. Sí, se rota entre compañeros de forma habitual.

D. Sí, se cambia según lo considera el trabajador.

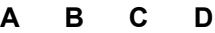

\section{Pregunta 26}

Ante la incorporación de nuevas tecnologías, nueva maquinaria y/o nuevos métodos de trabajo ¿se instruye al trabajador de seguridad para adaptarlo a esas nuevas situaciones?

A. No.

B. Sí, oralmente.

C. Sí, por escrito

D. Sí, oralmente y por escrito.

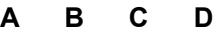

\section{Pregunta 27}

Pregunta 24

¿Usted como trabajador de seguridad recibe información suficiente sobre los resultados de su trabajo?

A. Se le informa de la tarea a desempeñar (cantidad y calidad)

B. Se le informa de los resultados alcanzados con relación a los objetivos que tiene asignados.

C. Se le informa de los objetivos alcanzados por la empresa.

D. Se le anima a participar en el establecimiento de metas.
¿Qué tipo de relaciones son las habituales en la empresa? colaboración para el trabajo y relaciones personales positivas.

B. Relaciones personales positivas, sin relaciones de colaboración.

C. Relaciones sólo de colaboración para el trabajo

D. Ni relaciones personales, ni colaboración para el trabajo..

\section{A B C}

\section{A $\quad$ B $\quad$ C $\quad$ D}


Pregunta 28

De los problemas que existen en un departamento, sección... ¿está siendo culpada alguna persona en concreto?

A. Sí.

B. No.

A B

\section{Pregunta 29}

¿Han aumentado las bajas de origen psicológico de larga duración en la plantilla?

A. Sí.

B. No.

\section{Pregunta 30}

¿Hay alguna persona que está siendo aislada, ignorada o excluida del grupo en virtud de características físicas o personales?

A B
A. Sí.

B. No.

A B 